\title{
APPUNTI PER LA PROSPEZIONE SISMICA DELLE ZONE CON RIFLESSIONI SCARSE O INSUFFICIENTI
}

\author{
Camillo Contini
}

1. Premesse. - Esistono delle zone molto vaste, in Italia come in tutte le parti del mondo, le quali danno luogo a riflessioni delle onde sismiche saltuarie e scarse tali da rendere incerta o impossibile la ricostruzione della tettonica degli strati profondi mediante i rilievi sismici a riflessione.

Negli Stati Uniti, secondo le più recenti informazioni $\left({ }^{1}\right)$, risulta che di tutta l'area coperta da strati sedimentari (circa il 75\% dell'area totale), ove le riflessioni sono possibili, per il $17 \%$ si hanno riflessioni buone e sicure, per il $28 \%$ riflessioni deboli e di scarsa attendibilità e per ben il $55 \%$ riflessioni saltuarie o assenti e in ogni caso incerte. Per l'Italia le conoscenze sono ancora troppo scarse per poter calcolare percentuali del genere: sappiamo di certo però che le zone in cui le riflessioni risultano scarse o insufficienti non possono che essere molto estese. Per esempio, sappiamo che le riflessioni sono

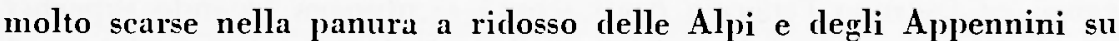
una fascia di una diecina di chilometri di larghezza, nelle zone di Casalpusterlengo e di Ferrara, nella Pianura Veneta, nelle Marche e nella Valle Bradanica e che sono insufficienti o assenti sull'Appennino, sul versante padano, nella zona a Sud di Padora, nella zona del Delta Padano a oriente di Copparo, sulla "Dorsale ferrarese ", nella zona a Nord di Milano fra il Ticino e l'Adda, ecc.

Queste zone di riflessioni scarse o insufficienti possono essere di interesse per le ricerche minerarie esattamente quanto quelle di riflessioni buone, considerato che, in generale, le cause che incidono sull'attendibilità delle riflessioni sono dorute semplicemente alle caratteristiche elastiche dei terreni prossimi alla superficie del suolo e non agli strati profondi: rediamo allora l'utilità pratica di adottare tutti gli accorgimenti che valgano a mirliorare $i$ dati ove questi siano scarsi e ottenere almeno qualche elemento indicativo quando le riflessioni siano del tutto assenti.

Le riflessioni, quali risultimo dai sismogrammi di rilievo, possono 
riuscire scarse o insufficienti per due ragioni diverse: a) perché le onde sismiche prodotte dagli scoppi sono di intensità insufficiente; $b$ ) perché le onde di fondo, i cosidetti " Disturbi ", hanno notevoli ampiezze per cui, sovrapponendosi alle riflessioni utili, rendono queste irriconoscibili.

Per aumentare l'intensità delle onde si possono adottare gli accorgimenti: 1) di aumentare l'energia delle onde generate dagli scoppi aumentando le cariche delle mine e approfondendo opportunamente i pozzetti delle mine stesse; 2 ) di aumentare la sensibilità delle registrazioni e di aumentare l'energia delle onde riflesse che agiscono sui sismografi.

Per ridurre invece $i$ disturbi in modo da rendere reperibili le riflessioni utili si possono adottare questi altri accorgimenti; 1) di impiegare dei filtri particolarmente adatti alle onde riflesse della zona in rilievo con bande il più possibile ridotte e aumentare il numero e le distanze dei sismografi collegati ad ogni filo di registrazione; 2) adottare il sistema delle registrazioni per (Trascinamento ).

Nei numeri che seguono vedremo in dettaglio l'utilità e la convenienza dell'applicazione di tali accorgimenti nell'esecuzione pratica dei rilievi di prospezione mineraria.

2. Energia degli scoppi. - In un mezzo elastico indefinito omogeneo ed isotropo l'energia degli scoppi si propaga secondo superfici d'onda sferiche col centro nei punti di scoppio; al crescere dei raggi delle superfici d'onda crescono le superfici su cui l'energia si deve distribuire: l'energia per unità di superficie delle onde diminuirà quindi coi quadrati dei raggi delle superfici d'onda. Poiché l'energia a sua volta ̀̀ proporzionale al quadrato delle ampiezze, avremo in definitiva che le ampiezze stesse diminuiscono linearmente al crescere delle distanze, ossia che

$$
\mu=\frac{\mu^{\prime}}{x},
$$

ove $x$ siano le distanze degli scoppi, $\mu$ le ampiezze delle onde alle distanze $x \in \mu^{\prime}$ le ampiezze alla distanza unitaria.

Ma le ampiezze delle onde alle stesse distanze dagli scoppi sono direttamente proporzionali alle radici quadrate delle cariche delle mine $\left({ }^{2}\right)$; se allora indichiamo queste con $q$ e con $\mu_{0}$ le ampiezze relative alle cariche unitarie alle distanze unitarie abbiamo 


$$
\mu^{\prime}=\mu_{\mathrm{o}} \sqrt{q},
$$

e quindi, per la [1],

$$
\mu=\mu_{o} \frac{\sqrt{q}}{x} .
$$

Vediamo da quest'ultima che per ottenere una data ampiezza « delle onde alle diverse distanze $x$ dagli scoppi dobbiamo variare le cariche $q$ in ragione dei quadrati delle distanze $x$ stesse.

In effetto, i terreni che interessano la prospezione mineraria sono tutt'altro che omogenei ed isotropi e ammettono dei valori delle velocità di trasmissione delle onde variabili colle profondità: avremo quindi delle formole di variazione delle ampiezze più complesse di quelle sopra considerate. A titolo d'esempio, noi abbiamo trovato, per le ampiezze minime necessarie per le registrazioni, nei rilievi a rifrazione delle argille delle zone di Firenzuola-Pietramala e della Valle Bradanica, rispettivamente le equazioni

$$
\begin{gathered}
q=2 \cdot 10^{-6} x^{2}+8 \cdot 10^{-3} x, \\
q=\frac{6}{5} 10^{-6} x^{2}+\frac{19}{5} 10^{-3} x,
\end{gathered}
$$

$x$ in metri e $q$ in chilogrammi di dinamite G.D.I.

Quando a poca profondità esistessero degli strati con velocità di trasmissione elevata, orizzontali o con piccola inclinazione, l'energia rifratta alla superficie del suolo, almeno da una certa distanza in poi, risulta funzione pressoché lineare delle distanze stesse: ciò spiega come in generale nei rilievi a rifrazione risultino, invece delle [3] e [4], delle equazioni lineari in $q \mathrm{e}$ in $x\left({ }^{3}\right)$.

Nei rilievi a riflessione i raggi sismici raccolti dai sismografi si trasmettono trasversalmente agli strati del sottosuolo, i quali, date le differenziazioni delle relative caratteristiche elastiche, riflettono e assorbono i raggi con proporzioni estremamente variabili da strato a strato. Non vi è dubbio però che in media si abbiano delle leggi per le ampiezze delle onde riflesse al suolo del tipo della [3] o delle [4], ove con $x$ siano indicate le lunghezze dei percorsi dei raggi registrati.

A parità di peso delle cariche delle mine l'energia sismica trasmessa al suolo risulta pressoché proporzionale al potere dirompente dell'esplosivo usato. Per i più comuni esplosivi disponibili in Italia i 
poteri dirompenti, posto 100 quello della nitroglicerina, sono approssimativamente i seguenti:

Gelatina (trinitroglicerina) . . . . . . . . $\quad$. 100

Gomma A $(92 \%$ gelatina, $8 \%$ cotone collodio $)$. . . . . 87

Gomma B $(88 \div 89 \%$ gelatina, $11 \div 12 \%$ nitrato sodico, assorbenti) . . . . . . . . . . . . 73

Gomma G.E.O. (83 $\div 84 \%$ gelatina, $16 \div 17 \%$ nitrato sodico, as-

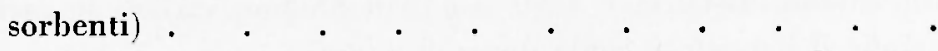

Dinamite $\mathrm{N}^{\circ} 1$, militare $(75 \%$ gelatina, $25 \%$ nitrato sodico, as-

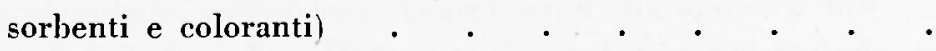

G.D.I $(62,5 \div 63 \%$ gelatina, $37 \div 37,5 \%$ nitrato sodico, assorbenti e coloranti) . • . . . . . . $\cdot$.

G.D.II $(43,5 \div 44 \%$ gelatina, $56 \div 56,5 \%$ nitrato sodico, assor-

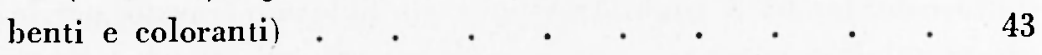

Tritolo (trinitrotoluolo) . . . . . . . . . . $\quad . \quad 50$

Acido picrico (trinitrofenolo) . . . . . . . . 53

Fulmicotone . . . . . . . . . . . . . 70

Fulminato di mercurio . . . . . . . . . $\quad$. 25

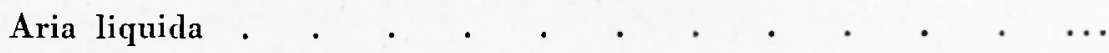

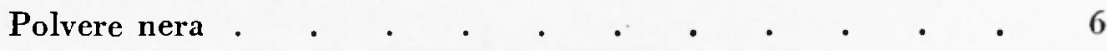

Quando le ampiezze $\iota_{0}$ fossero relative alle cariche unitarie di gelatina (trinitroglicerina) evidentemente possiamo porre, per la [3],

$$
\mu=\mu_{0} \frac{\sqrt{\varepsilon}}{10} \frac{\sqrt{q}}{x},
$$

essendo indicato con $\varepsilon$ il potere dirompente degli esplosivi usati per gli scoppi.

Come si sa, l'energia sismica irradiata dagli scoppi nel terreno è funzione anche delle caratteristiche elastiche delle rocce a contatto colle mine. Queste rocce devono essere consolidate e con velocità di trasmissione delle onde approssimativamente uguale a quella media dei primi strati ove i raggi debbono trasmettersi. L'esperienza diretta indicherà quali saranno le profondità dei pozzetti da mina cui corrispondono i rendimenti ottimi degli scoppi. Sempre però si dovrà sparare sotto il cosidetto "Strato aerato superficiale ), cui corrispondono delle velocità di trasmissione dell'ordine di $1 / \underline{2} \div \overline{1} / 3$ di quelle degli strati consolidati immediatamente sottostanti. Le potenze di questo stratu nella Valle Padana possono arrivare fino a 30 metri in alcune 
zone; in media però sono dell'ordine dei $10 \div 20$ metri, e variano da punto a punto in modo abbastanza regolare per permettere una sufficientemente accurata determinazione durante l'esecuzione dei normali rilievi $\left({ }^{4}\right)$.

Per perforare i pozzetti colle profondità volute nelle zone franose con ghiaie grossolane, ove le normali perforatrici automontate a rotazione si dimostrano inadatte, noi abbiamo costruito delle speciali perforatrici a percussione (5).

Scoppiando sotto l'aerato si ha anche il vantaggio di evitare la dispersione dell'energia per effetto della riflessione alla superficie limite inferiore dello stesso. Viceversa, se si scoppia sotto l'aerato vicino alla superficie limite, all'energia diretta degli scoppi si somma quella riflessa dalla stessa superficie limite. Come è risaputo, le ampiezze delle onde riflesse alle superfici limiti fra due mezzi con velocità di trasmissione $v^{\prime}$ e $v_{\mathrm{s}}$ sono approssimativamente date, in percento di quelle delle onde incidenti, supposta costante la densità, dalla

$$
\eta=\frac{v_{s}-v^{\prime}}{v_{s}+v^{\prime}} .
$$

Se in questa sostituiamo i valori dei terreni superficiali e dell'aerato quali si hanno nella media Valle Padana, $v^{*}=550 \mathrm{~m} / \mathrm{s}, v_{\mathrm{s}}=1700 \mathrm{~m} / \mathrm{s}$, otteniamo $\eta=\sim 1 / 2$ : vediamo allora che per la sola riflessione alla superficie base dell'aerato si ha una differenza di circa il $25 \%$ delle ampiezze scoppiando entro o sotto lo stesso aerato.

Le lunghezze delle cariche non devono assolutamente superare quella di $1 / 0$ delle lunghezze d'onda medie delle bande dei filtri usati nelle registrazioni, onde evitare dannose deformazioni dei fronti d'onda e interferenze tali da ridurre sensibilmente l'energia sismica immessa nel suolo. Risulıa allora la condizione, indicando con $f_{c}$ le frequenze medie delle bande dei filtri e con $\gamma$ le lunghezze delle cariche,

$$
\gamma<\frac{1}{10}-\frac{v_{s}}{f_{c}} .
$$

Nel caso delle cariche di tritolo compresso col diametro di $60 \mathrm{~m} / \mathrm{m}$, largamente usate in Italia, si hanno delle lunghezze di circa $25 \mathrm{~cm}$ per ogni chilogrammo di esplosivo e quindi risultano i limiti per le cariche $q$, posto $v_{\mathrm{s}}=1700 \mathrm{~m} / \mathrm{s}$, di $34,17,12$ e $8,5 \mathrm{~kg}$ rispettivamente per le frequenze di $20,40,60$ e $80 \mathrm{~Hz}$.

Quando le cariche $q$ dovessero essere maggiori di quelle che ri- 
sultano dalle lungheaze limite fissate dalla [7] si aumenteranno i diametri delle cariche oppure si ripartiranno le cariche in due o più pozzetti. Fra queste soluzioni sarebbe da sceglicrsi, secondo le ricerche di Shape $\left({ }^{6}\right)$, la prima quando si utilizzassero frequenze $f_{1}$ molıo basse e quindi sarebbe conveniente cercare di aumentare la percentuale dell'energia trasmessa al suolo con onde pure di bassa frequenza. In proposito dobbiamo però osservare che per le maggiori cariche l'energia sismica sviluppata dagli scoppi, al crescere delle cariche, aumenta in generale meno rapidamente di quanto vuole la [2] e quindi che al di sopra di certi valori di $q$, da stabilire zona per zona, conviene adottare il sistema dei pozzetti multipli ('). Con questo sistema, come vedremo in seguito, si raggiunge in pratica anche lo scopo di diminuire l'ampiezza delle onde di fondo per rispetto a quella delle riflessioni utili; si ha però l'inconveniente del dispendio non indifferente della perforazione di numerosi pozzetti, i quali, naturalmente, devono raggungere tutti la profondità di rendimento oltimo degli scoppi.

L'innescamento delle cariche dev'essere sempre fatto dalla parte superiore; ciò per due ragioni: 1) perché, risultando in generale più energici gli effetti delle esplosioni nella direzione di propagazione dell'accensione, si abbia una maggiore energia sismica nella direzione utile; 2) perché risultino ridotti gli sfasamenti fra le onde sismiche dipartentesi dagli estremi inferiori e superiori delle cariche.

Infine notiamo che, in generale, il rendimento in energia sismica degli scoppi aumenta colla profondità degli scoppi stessi. Vedremo in seguito come, potendo perforare dei pozzetti da mina molto profondi, si possano anche ripartire le cariche in due o più punti diversi alla distanza l'uno dall'altro di pochi metri o addirittura di una intera lunghezza d'onda media della banda del filtro usato in modo da ottenere gli stessi effetti utili che abbiano visto per i pozzetti multipli.

Naturalmente le nostre considerazioni non escludono l'applicazione di tutti quegli accorgimenti e quelle norme che risultano dalla lunga esperienza dei nostri artificieri sulla ricerca delle profondità ottime di scoppio, sulla confezione delle mine, sul collocamento e l'innescamnto delle stesse, sulla "Borratura », ecc. e che costituiscono oramai un'arte assai complessa, data anche la dettagliata regolamentazione da parte delle autorità di Pubblica Sicurezza $\left({ }^{8}\right)$.

Il sistema di scoppiare in aria libera da noi esperimentato nella zona di Lodi ha dato risultati di gran lunga inferiori a quelli otte- 
nuti scoppiando nei pozzelti solto lo strato aerato. Può darsi però che in altre zone possa avvenire viceversa. A nostro parere riuscireblue utilissimo nella zona a Nord di Milano fra il Ticino e l'Adda ove non è possilbile oltrepassare, né colle perforatrici a rotazione né con quelle a percussione, lo strato aerato, composto di ghiaie grossolane con alternanze di conglomerati $\left({ }^{8}\right)$.

3. Postazione dei sismografi sul terreno. - Per comodità si tende, nell'esecuzione pratica dei rilievi, ad usare pochi sismografi e a poggiare gli stessi sul terreno senza nessuna preparazione. Nelle zone ove le riflessioni sono facili e sicure non vi è ragione, evidentemente, per operare altrimenti ma non così nelle zone di difficile rilievo, ove l'energia riflessa risulta insufficiente. In queste zone lisogna prima di tutto fare in modo che la sensibilita delle misure sia di un ordine di grandezza di poco inferiore a quello dei "Microsismi " e delle piccolissime vilurazioni provocate dall'attività umana.

Tale sensiljilità in genere si raggiunge soltanto inserendo più sismografi sui diversi fili registratori e poggiando i sismografi stessi sul terreno consolidato, sotto quello normalmente coltivato, con scavi di $20 \div 30 \mathrm{~cm}$ di profondità.

Sovente avviene però che, malgrado si sia raggiunta tale sensiljilità limite, ancora risulti insufficiente o assente l'energia riflessa dagli strati profondi. Allora si metteranno in atto gli accorgimenti utili per aumentare l'energia sismica trasmessa al suolo: aumento delle cariche, pozzetti multipli, approfondimento dei pozzetti, ecc.

Come ultima risorsa si potrà tentare l'esperimento di porre $\mathrm{i}$ sismografi al fondo di pozzetti, sotto la base dell'aerato. Questo esperimento riuscirà certamente molto costoso dato il gran numero di pozzetti da scavare: in generale però darà buoni risultati. Ciò per due ragioni: perché sotto l'aerato l'energia in arrivo risulta aumentata della percentuale riflessa alla base dello stesso aerato anziché diminuita e perché l'aerato assorbe sempre delle percentuali fortissime dell'energia che arriva ad esso.

$\mathrm{E}$ da tenere presente in proposito che in generale le zone con riflessioni scarse o insufficienti hanno l'aerato molto potente composto di terreni pochissimo consolidati con velocità di trasmissione particolarmente bassa. Per esempio nella pianura a ridosso dell'Appennino si ha una potenza di $25 \div 30$ metri e una velocità di $400 \div 450$ $m$ 's. 
Ponendo i sismografi nei pozzetti si può anche eliminare, come è evidente, l'influenza delle irregolaritì della base dell'aerato e della topografia superficiale nelle zone alquanto frequenti ove queste irregolarità sono notevoli.

Le tubazioni dei pozzetti dei sismografi debbono essere leggere in modo che non possono vibrare indipendentemente dal terreno circostante. I sismografi debbono essere premuti contro il fondo dei pozzetti oppure annegati in acqua e devono essere tenuti verticali da speciali apofisi.

4. Filtri. - I periodi $T$ delle onde riflesse aumentano al crescere delle distanze $\lambda$ percorse approssimativamente secondo la formola $\left({ }^{10}\right)$

$$
T^{2}=T_{0}^{2}+\frac{1}{10} \lambda,
$$

ove $T_{\mathrm{o}}$ è il periodo delle onde all'origine, $T$ e $T_{\mathrm{o}}$ in millisecondi e $\lambda$ in metri: da ciò l'utilità di operare con filtri di frequenza molıo basse quando si debbono rilevare degli strati molto profondi. Le frequenze molto basse risultano anche adatte per il rilievo delle zone ove l'energia riflessa è scarsa, in quanto, come si sa, le onde lunghe sono in generale più persistenti delle corte e sorpassano più facilmente le eterogreneità e le discontinuità del terreno.

Operando colle massime lunghezze d'onda, riusciranno molto sensibili gli effetti della djffrazione delle onde delle eterogeneità del terreno e delle eventuali discontinuità delle superfici riflettenti e bisognerà adoltare tutti gli accorgimenti che altrove abbiamo visto nella lettura delle riflessioni sui sismogrammi e nelle elaborazioni delle stesse $\left({ }^{11}\right)$.

5. Selezione delle onde riflesse. - Ottenute in un modo qualsiasi le ampiezze delle onde riflesse sufficienti per le registrazioni, dobbiamo ancora fare in modo che le "Riflessioni", ossia le onde riflesse dalle superfici limite degli strati, utili per il calcolo degli elementi geometrici delle stesse, risultino chiaramente leggibili sui sismogrammi. Raggiungiamo lo scopo se riduciamo sufficientemente le ampierze delle onde di fondo in rapporto a quelle delle riflessioni utili.

Le onde di fondo, per la loro stessa natura, hanno delle fasi che per rispetto a quelle delle riflessioni si possono ritenere caotiche. Ora, se noi colleghiamo in serie molti sismografi, $M_{1}, M_{2}, \ldots \ldots . M_{n}$, ognuno dei quali sollecitato da onde con fasi a caso ma colla stessa fre- 
quenza, otteniamo le ampiezze, secondo i calcoli delle probabilità fatte da Lord Rayleigh per le onde luminose,

$$
u=\sqrt{\mu_{1}^{2}+\mu_{2}^{2}+\cdots \cdots+\mu_{n}^{2}},
$$

essenclo $\mu_{1}, \mu_{2}, \ldots . . . \varphi_{n}$ le ampiezze delle onde d'uscita dei diversi sismografi. Posto, per semplicita, $\mu_{1}=-\mu_{2}=\ldots . .=\iota_{12}$, risulta che le ampiezze dei disturbi somma di quelli dei diversi sismografi sono uguali a quelli dei singoli sismografi moltiplicati per la radice quadrata del numero $n$ degli stessi.

Le onde costituenti le riflessioni utili invece si possono considerare fra loro in fase per zone anche molto vaste. Supposto allora che le onde d'uscita dei sismografi $M_{1}, M_{2, \ldots . .} M_{n}$ siano pressoché in fase e di uguale ampiezza, otteniamo delle ampiezze somma uguali alle ampiezze dei singoli sismografi moltiplicate semplicemente per il numero n. dei sismografi stessi.

Dal confronto dei risultati dei due casi risulta che in effetto, colla postazione di $n$ sismorrafi in serie, le ampiezze delle onde di fondo sono diminuite, per rispetto a quelle delle riflessioni utili, del rapporto da 1 a $1 / n$, ossia di $1 n$.

L'estensione delle zone entro cui le onde delle riflessioni si possono considerare in fase i evidentemente funzione delle pendenze delle superfici riflettenti e delle lunghezze d'onda dei filtri utilizzati; sarà minima nella direzione di massima pendenza degli strati e massima nella direzione perpendicolare. Indicando con $\Delta_{r_{x}}$ l'incremento massimo dei tempi delle riflessioni nella direzione di massima pendenza degli strati per le lunghezze $2 l$ dei profili dei sismografi, con $T$. le lunghezze medie rielle oncle delle hande dei filtri usati (misurate in tempi sui sismogrammi), con $j$ le distanze dei sismografi dai centri dei gruppi relativi fissati sul terreno secondo gli schemi di rilievo adoltati, con o le direzioni degrli spostamenti dei sismografi dagli stessi centri misurate rispello alla direzione di massima pendenza derli strati e con tor le differenze dei valori del move out normale corrispondenti ai punti di postazione dei sismografi e ai centri dei gruppi relativi possiamo porre

$$
j<\frac{2 l}{\Delta \tau_{\mathrm{x}} \cos \delta}\left(\frac{T_{\mathrm{c}}}{30}+\Delta \delta \tau_{\mathrm{n}}\right),
$$


nell'ipotesi che gli sfasamenti massimi fra i sismografi e i centri dei gruppi relativi non superino $i$ valori di $T_{\mathrm{c}} 30$, ossia che gli sfasamenti massimi fra i sismografi degli slessi gruppi non superino i valori di $T_{\mathrm{c},} 15$.

Le distanze minime fra i diversi sismografi degli stessi gruppi oltre le quali le fasi delle onde di fondo risultano sensibilmente indipendenti sono funzione delle particolari condizioni tetloniche e litologiche degli strati riflettenti e di quelli attraversati dai raggi e delle lunghezze d'onda utilizzate per le registrazioni. Noi siamo convinti che preralentemente le onde di fondo siano attribuibili ai fenomeni della diffrazione che insorgono dalle eterogeneitì dei terreni, dalle rotture e discontinuità degli strati, dai piegamenti con grande curvatura, ecc.; se ciò è vero, essendo l'intensità dei fenomeni pressoché proporzionale a $T_{\text {, }}$, arremo la condizione per $\mathrm{i}$ valori $i$ delle distanze fra sismografo e sismografo

$$
i>\xi \sqrt{T_{\mathrm{c}}},
$$

ove $\xi$ sia una costante da determinare sperimentalmente. Per $T_{\mathrm{c}}$ espressi in millisecondi ed $i$ in metri risultereblero in pratica dei valori per $\xi$ dell'ordine di $3 \div 5$ unitì e anche magrgiori.

Data la condizione [11], o altra simile meglio rispondente ai dati sperimentali, in generale risulterà la possibilita di disporre sugli allineamenti dei profili non più di $2 \div 3$ sismografi per gruppo, anche per le maggiori lunghezze dei profili possiljili in pratica. Onde poter, allora, utilizzare un numero di sismografi sufficiente per ridurre sensilbilmente $\mathbf{i}$ disturlbi bisognerà disporre $i$ sismografi stessi secondo allineamenti normali ai profili: le distanze dai profili base potranno anche salire fino a una cinquantina di metri, da una parte e dall'altra, quando si arrà ragione di ritenere continui gli strali riflettenti, naturalmente, sempre che siano soddisfatte le condizioni [10] e [11]. Come regola conviene disporre i sismografi in modo simmetrico rispetto ai profili base.

Un sistema di riliero che può rinscire molto semplice per gli operatori in campagna, quando si debloono usare molti sismografi, è quello di disporre i sismografi stessi secondo due profili paralleli, distanti fra loro anche di un centinaio di metri, ognuno con gruppi di sismografi il più possibile numerosi, di collegare in serie $\mathrm{i}$ gruppi corrispondenti alle medesime normali e di scoppiare nel mezzo dei profili sulla mezzaria comune. 
Infine si possono raddoppiare praticamente $i$ sismografi in serie sui fili di registrazione, qualunque sia la disposizione adottata, collegando in serie a due a due i gruppi; operando in tale modo, poiché in effetto i grruppi vengrono ridotti alla metì, si può regristrare ogni scoppio con due filtri diversi sui medesimi sismogrammi, effettuare, cioc, le cosiddette "Registrazioni in duale".

Se, come noi crediamo, le onde di fondo sono dovute ai fenomeni della diffrazione, evidentemente otterremo i medesimi effetli dei sismografi multipli collegati in serie adottando i pozzetti multipli e gli scoppi multipli nei pozzetti profondi, di cui abbiamo detto al n. 2 : $n$ pozzetti o $n$ scoppi equivarrebbero a $n$ sismografi per gruppo, naturalmente quando per le distanze fossero verificate le stesse condizioni [10] e [1]]. Come è evidente, gli accorgimenti dei sismografi e dei pozzelti o degli scoppi multipli si possono applicare contemporaneamenle con effetti utili.

Applicando l'accorgrimento dei sismografi mulıpli si ha la possibilita di determinare le potenze dello strato aerato superficiale, mediante i dati dei normali scoppi di rilievo, soltanto in corrispondenza ai sismografi più vieini agli scoppi dei vari gruppi; in compenso però, mediando molti valori, si elimina la maggior parte delle influenze delle irregolarità di andamento delle potenze stesse.

6. Trascinamento delle onde. - Qualunque siano le disposizioni adottate per i sismografi dei profili di rilievo avremo sempre un miglioramento delle riflessioni, almeno nella loro apparenza, coll'adozione del sistema di registrazione del "Trascinamento delle onde ), chiamato con "Mixing ) dagrli americani.

Le onde si dicono "Trascinate" quando nella registrazione sono composte le une colle altre in modo da ridurre le iniziali differenze di fase e di ampiezza e dare luogo a riflessioni sui sismogrammi allineate e appariscenti, facilmente rilevabili nelle loro caratteristiche di tempo.

Cogli strumenti moderni il trascinamento è realizzato nel seguente modo:

1) la corrente che ogni gruppo di sismografi invia al registratore viene da questo filtrata, amplificata e infine suddivisa in due parti $i_{1}$ e $i$, tali per cui

$$
i_{2}=\rho\left(i_{1}+i_{2}\right)=\frac{\rho}{1-\varrho} i_{1}
$$


ove $\varrho$, "Costante di trascinamento », fissata a volontà, minore o tutt'al più uguale a $1 / \%$;

2) la corrente $i_{1}$ dei gruppi di sismografi estremi dei profili viene inviata ai galvanometri di registrazione corrispondenti e quindi registrata sui sismogrammi; la corrente $i_{2}$ invece viene posta in parallelo $o$ in serie con quella $i_{1}$ dei gruppi adiacenti e inviata ai galvanometri corrispondenti, il $2^{\circ}$ e $1^{\prime} 11^{\circ}$, per la registrazione sui sismogrammi;

3) ugualmente la corrente $i$, dei gruppi $2^{\prime \prime}$ e $11^{\circ}$ viene messa in parallelo o in serie con quella $i_{1}$ dei gruppi adiacenti e inviata ai galvanometri corrispondenti, il $3^{\circ}$ e il $10^{\circ}$, per la registrazione; la corrente $i_{2}$ degli stessi gruppi invece viene posta in parallelo o in serie con quella $i_{1}$ dei gruppi adiacenti, e così di seguito;

4) la corrente $i$, dei gruppi $6^{\circ}$ e $7^{\circ}$ viene annullata, chiudendo i circuiti su adeguate resistenze $o$ in altro modo qualsiasi.

I circuiti sono studiati in modo da rendere praticamente insensibili le influenze dei vari gmppi di sismografi su quelli adiacenti verso l'esterno dei profili (così che il trascinamento avvenga soltanto dai gruppi esterni verso quelli esterni e non viceversa) e su quelli successivi agli immediatamente adiacenti verso l'interno.

Effettuando le registrazioni col trascinamento delle onde o, come si dice, "In trascinamento", col sistema sopra descritto, risultano le seguenti variazioni delle caratteristiche delle riflessioni quali appaiono sui sismogrammi :

a) mentre i tempi delle onde dei fili estremi risultano corrispondenti a quelli effettivi delle onde pervenute ai sismografi estremi dei profili quelli delle onde dei fili $2^{\circ}$ e $11^{\circ}$ risultano variati mediamente di circa $1 / 2 \delta \tau \varrho /(\mathrm{I}-\varrho)$, essendo $\delta \tau$ l'incremento dei tempi delle riflessioni corrispondente alla distanza $\lambda$ fra $\mathrm{i}$ centri dei gruppi dei sismografi, circa

$$
\delta \tau=\frac{\lambda}{2 l} \Delta \tau_{\boldsymbol{\alpha}} \cos \delta
$$

uguali variazioni si hanno per i fili successivi dal $2^{\circ}$ al $6^{\circ}$ e dall' $11^{\circ}$ al $7^{\circ}$; le variazioni sono sempre nel senso di una diminuzione delle differenze dei tempi rispetto a quelli corrispondenti ai fili estremi;

b) gli allineamenti delle riflessioni utili sono variati in quanto fra i fili estremi e gli adiacenti risultano gli incrementi di fo $(1-0)$, fra i fili dal $2^{\circ}$ al $6^{\circ}$ e dall' $11^{\circ}$ al $7^{\circ}$ gli incrementi di $\delta r$ e fra i fili $6^{\circ}$ e $7^{\circ}$ l'incremento di $\delta \tau(1+2 \varrho)$; 
c) nei fili dal $2^{\circ}$ al $6^{\circ}$ e dall' $11^{\circ}$ al $7^{\circ}$ le onde di fonclo accusano delle variazioni di fase da filo a filo ridotte, rispetto alle effettive, in media della frazione $\% / 2 /(1-\rho)$ e delle ampiezze ridotte in proporzione delle differenze delle fasi effettive: ciò equivale in pratica a una riduzione molto sensibile delle influenze nocive delle onde di fondo stesse e quindi a rendere gli allineamenti delle riflessioni più regrolari e più facilmente rilevabili dai sismogrammi, quando le distanze i. fra i centri dei vari gruppi soddisfano le [10] e [11].

Questo sistema di trascinamento presenta molti inconvenienti, fra cui quello di alterare gli allineamenti di cui sopra abbiamo detto; esso però può essere applicato alternativamente o contemporaneamente col sistema di registrazione con gruppi indipendenti senza richiedere nessuna variazione dei sismografi sul terreno o del loro allacciamento coi cavi di collegramento agli strumenti di registrazione.

Nelle zone di rilievo molio difficile in grenerale le registrazioni con gruppi indipendenti non danno risultati praticamente utilizzaloili e non vengono effettuate. In lali casi risulta superflua la possibilità di passare rapidamente da un sistema all'altro di registrazione e conviene adotlare un sislema di trascinamento più razionale, il quale non dia luogo ai difetti di cui sopra abbiamo detto.

Lo scrivente propone di adottare il sistema che si olliene da quello testé descritto ponendo $0=1 / 2$, rimendo in uno solo i gruppi $6^{\circ}$ e $7^{\circ}$, sostituendo i gruppi estremi con due gruppi separati e annullando le correnti $i$ dei gruppi estremi nello stesso modo adottato per annullare le correnti in dei gruppi $6^{\circ}$ e $7^{\circ}$.

Con lale sistema evidentemente si deblono usare 13 gruppi di sismografi anziché 12 e si debhono misurare le lunghezze dei profili fra i centri delle coppie estreme dei sismografi; le onde registrate dai diversi fili risultano esallamente medie fra quelle dei gruppi successivi dei sismografi, di uguale attendibiliti e ugualmente selezionate dalle onde di fondo; gli incrementi tolali delle riflessioni risultano ugruali a quelli che si avrebhero coi gruppi indipendenti posti in corrispondenza alle mezzarie delle coppie estreme, sebbene con precisione magrwiore, in media pressoché doppia; gli incrementi parziali non vengono diversamente variati e quindi le riflessioni risultano perfettamente allineate, simili a quelle che si ottengono coi sismografi indipendenti. Evidenti sono gli accorgimenti che si possono adottare per rendere il sistema rapido e sicuro nell'esecuzione pratica dei rilievi di campagna. 
Si dimostra facilmente che gli stessi risultati si possono ottenere mediante le registrazioni senza trascinamento duplicando i sismografi dei gruppi intermedi dal $2^{\circ}$ al $12^{\circ}$ e collegando ai fili estremi i sismografi del $1^{\circ}$ gruppo con quelli di una serie del $2^{\circ}$ e i sismografi dell'ultimo gruppo con quelli di una serie del $12^{\circ}$, ai fili $2^{\prime \prime}$ e $11^{\circ}$ le serie restanti dei gruppi $2^{\circ}$ e $12^{\circ}$ con una serie dei gruppi adiacenti $3^{\circ}$ e $11^{\prime \prime}$, e cosi di seguito.

L'attendililità delle riflessioni registrate in trascinamento coi sistemi descritti, per $\varrho=1 / 2$, è evidentemente un po' minore di quella delle riflessioni registrate coi circuiti indipendenti a parità di peso quale appare dai sismogrammi: in pratica però $\grave{e}$ sempre tale per cui le riflessioni, anche se di peso molto ridotto, risultano sicuramente riferibili a onde riflesse da orizzonti profondi quando appaiono in sequenze di due o più profili.

Per agevolare il calcolo deile potenze dello strato aerato superficiale gli strumenti moderni in generale registrano $i$ tempi delle onde dirette coi gruppi di sismografi indipendenti e automaticamente si dispongono in trascinamento per la registrazione delle onde riflesse successive.

Milano - febbraio 1953.

\section{RIASSUNTO}

Sono discussi gli accorgimenti che si debbono adottare per rendere possibile la prospezione sismica delle zone ove le riflessioni, ottenute colla procedura normale, risultano scarse o insufficienti. In particolare vengono discussi $i$ metodi per aumentare l'energia delle onde immesse al suolo e quelli per diminuire le ampiezze dei disturbi in confronto alle ampiezze delle riflessioni utili. Infine viene discusso il sistema di registrazione col "Trascinamento" delle onde.

\section{SUMMARY}

Te discuss here the precautions that must be adopted in order to carry out seismological prospecting on zones uhere the reflections as obtained by ordinary methods are scarce or insufficient. In particular, methods are discussed for augmenting the energy of the 
wave sent into the soil and for diminishing the amplitude of the disturbances in comparison with the amplitude of the aseful reflections. At the end we discuss the system of registration with "Betrayal" of the raves.

\section{BIBLIOGRAFIA}

(1) P. L. Lyovs: A seismic reflection quality map of the United States. Geophysies, vol. XVI, n. 3, 1951.

(2) F. Rixwnx: Zur experimentellen Seismik. Zeitschrift für Geophysik, vol. $\mathrm{XI}, \mathrm{n}, 4$ e 5,1935 .

(3) C. A. Ifallayd : Geophysical Exploration. Prentice Hall, New York, 1951, palg. 493.

(1) M. Sigvivi: La determinazione dello strato aerato superficiale nelle prospezioni sismiche a riflessione. Atti del VII Conv. Niz. Metano e Petrolio, 1952, vol. I, pag. 417.

(5) C. Contini: I pozzelti battuti nei rilievi sismici a riflessione. La Rivista Italiana del Petrolio, n. 225 e 226, 1952.

(6) J. H. Sirupe: The production of elastic waves by explosion pressure. Geophysics, vol. VII, n. 1, 1912.

(7) J. J. JakoskY: Exploration geophysics. Trija Publishing C. Los Angeles, 1950, pac. 855.

G. M. HanmerJan and J. T. Whettov: On the relationship beteveen seismic amplitude and charge of explosive fired in routine blasting operations. Geophysics, vol. XVII, n. $1,1952$.

(s) E. I. Du PoNt de. Nwwours and Co.: Blaster' handbook. Wilmington 98, Delaware, 1919.

C. BeLcruso: Gli esplosivi, con particolare riferimento a quelli da mina. agli innescamenti, alle polveri e cartucce da caccia e descrizione del loro impiego. Hoepli, Milano, 1952.

Ministero dell Interno. Direzione Generale della Pubblica Sicurezza: Regolamento per l'esecuzione del Testo Unico 18 giugno 1931-IX, n. 773 delle leggi di Pubblica Sicurezza (R. Decreto 6 magrio 1940.XVIII, n. 635 pubblicato nel supplemento ordinario alla "Cazzetta Ufficiale" del 26 giugno 1940-XVIII, n. 149), Istituto Poligrafico dello Stato. 1940.

(9) T. C. Poulten: The Poulher seismic method of geophysical exploration. Geophysics, vol. $\mathrm{XV}$, n. 2, 1950.

(10) B. Gutexberc: On some problems concerning the seismic field methods. Beiträge zur angewandten Ceophysik. 1937.

(11) C. Contwi: La diffrazione nei rilievi sismici a riflessione. Annali di Geofisica, vol. VI, n. 1. 1953. 